\title{
Membrane bile acid receptor TGR5 predicts good prognosis in ampullary adenocarcinoma patients with hyperbilirubinemia
}

\author{
MING-JENN CHEN ${ }^{1,2}$, YI-LING CHEN ${ }^{3}$, TZU-WEN WANG ${ }^{4}$, HUI-PING HSU ${ }^{4}$ and MING-DERG LAI ${ }^{5-7}$ \\ ${ }^{1}$ Department of Surgery, Chi Mei Medical Center, Tainan 710; Departments of ${ }^{2}$ Sports Management and \\ ${ }^{3}$ Senior Citizen Service Management, College of Leisure and Recreation Management, \\ Chia-Nan University of Pharmacy and Science, Tainan 717; ${ }^{4}$ Department of Surgery, \\ National Cheng Kung University Hospital, College of Medicine, National Cheng Kung University, Tainan 704; \\ ${ }^{5}$ Department of Biochemistry and Molecular Biology, ${ }^{6}$ Institute of Basic Medical Sciences, and \\ ${ }^{7}$ Center for Infectious Diseases and Signaling Research, College of Medicine, \\ National Cheng Kung University, Tainan 701, Taiwan, R.O.C.
}

Received March 12, 2016; Accepted May 5, 2016

DOI: $10.3892 / o r .2016 .5011$

\begin{abstract}
Bile acids are potential carcinogens in gastrointestinal cancer, and interact with nuclear and membrane receptors to initiate downstream signaling. The effect of TGR5 [also known as G protein-coupled bile acid receptor 1 (GPBAR1)] on cancer progression is dependent on the tissue where it is activated. In this report, the function of TGR5 expression in cancer was studied using a bioinformatic approach. TGR5 expression in ampullary adenocarcinoma and normal duodenum was compared by western blotting, reverse transcription polymerase chain reaction, and immunohistochemistry (IHC). High GPBARl gene expression was found to be an indicator of worse prognosis in gastric and breast cancer
\end{abstract}

Correspondence to: Dr Hui-Ping Hsu, Department of Surgery, National Cheng Kung University Hospital, College of Medicine, National Cheng Kung University, 138 Sheng-Li Road, Tainan 704, Taiwan, R.O.C.

E-mail: hphsu@mail.ncku.edu.tw

Abbreviations: AJCC TNM stage, American Joint Committee on Cancer tumor-node-metastases staging system; CA-125, cancer antigen-125; CA-199, cancer antigen-199; cAMP, cyclic adenosine monophosphate; CEA, carcinoembryonic antigen; COX-2, cyclooxygenase-2; EGFR, epidermal growth factor receptor; FXR, farnesoid X receptor; GCDC, glycochenodeoxycholate; GPBARl, $\mathrm{G}$ protein-coupled bile acid receptor 1; IHC, immunohistochemistry; IRS, immunoreactive score of Remmele and Stegner; JNK, c-Jun-N terminal kinase; MAPK, mitogen-activated protein kinase; PDGFR, platelet-derived growth factor receptor; RNS, reactive nitrogen species; ROS, reactive oxygen species; RT-PCR, reverse transcription polymerase chain reaction; STAT3, signal transducer and activator of transcription 3; TCDA, taurochenodeoxycholate

Key words: ampullary adenocarcinoma, bile acids, TGR5, GPBAR1, Kaplan-Meier plotter, PrognoScan, cBioPortal patients, and an indication of better prognosis in ovarian cancer patients. The level of GPBARl gene expression was higher in bile-acid exposed cancer than in other types of cancer, and was increased in well-differentiated ampullary adenocarcinoma. Negative, weak or mild expression of TGR5 was correlated with younger age, higher plasma level of total/direct bilirubin, higher plasma concentration of CA-125, advanced tumor stage and advanced AJCC TNM stage. The disease-specific survival rate was highest in ampullary adenocarcinoma patients with high TGR5 expression and high total bilirubin level. In summary, TGR5 functions as a tumor-suppressor in patients with ampullary adenocarcinoma and preoperative hyperbilirubinemia. Further study of the suppressive mechanism may provide a new therapeutic option for patients with ampullary adenocarcinoma.

\section{Introduction}

Carcinoma of the ampulla of Vater is the most common cancer of the small intestine and is commonly adenocarcinoma (1). The ampulla of Vater is located in the second portion of the duodenum, at the confluence of the common bile and pancreatic duct. Long-term exposure to bile acids is a possible reason for malignant transformation. Bile acids are potential carcinogens in gastrointestinal cancer, including gastric, esophageal and colon cancer, and cholangiocarcinoma (2). Incidences of intestinal metaplasia and gastric cardia cancer are increased in patients with gastroesophageal reflux disease (3). Treatment of squamous cell carcinoma cell lines of the esophagus with bile acids induces cell cycle progression and production of G1-regulating molecules (4). The secondary bile acids, deoxycholate and lithocholate, are the most well-known carcinogens in human colon cancer and are associated with the generation of reactive oxygen/nitrogen species (ROS/RNS) (5). The accumulation of ROS/RNS was found to cause oxidative DNA damage and further mutation in colon cancer (6). The conjugated bile acid glycochenodeoxycholate (GCDA) induced expression of cyclooxygenase 2 (COX-2) and genes that are 
related to cell proliferation in cholangiocytes (7). Another type of conjugated bile acid, taurochenodeoxycholate (TCDA), induced phosphorylation of epidermal growth factor receptor (EGFR) and its downstream signaling in a human cholangiocarcinoma cell line (8).

Bile acids interact with several types of nuclear receptors, including farnesoid X receptor (FXR), vitamin D receptor, pregnane $X$ receptor, and constitutive androstane receptor (9). Nuclear receptors are ligand-modulated transcription factors and regulate uptake, detoxification and secretion of bile acids. FXR is the main form of nuclear receptor of bile acids expressed in the gastrointestinal tract where it mediates homeostasis of bile acids, lipids and glucose. Expression of FXR is reduced in human colon cancer or Barrett's esophageal cancer. FXR functions as a tumor suppressor in colon, liver and esophageal cancer (10-12). The oncosuppressive roles of FXR in these cancers include suppression of proliferation and induction of apoptosis after exposure to bile acids. Bile acids also act as systemic hormones when interacting with membrane receptors, such as $\mathrm{G}$ protein-coupled bile acid receptor 1 (gene GPBAR1; also known as TGR5, M-BAR and BG37). Bile acid-dependent TGR5 activation is involved in the immunomodulatory properties of bile acids, synthesis of endothelial nitric oxide, and mitochondrial energy homeostasis $(2,9)$. In the normal physiologic condition, the functions of TGR5 include modulation of gallbladder filling, improvement of insulin sensitivity, maintenance of glucose homeostasis, and increased energy expenditure to attenuate diet-induced obesity $(13,14)$. The function of TGR5 is variable in different types of cancer (15). Expression of TGR5 is increased in the intestinal subtype of gastric adenocarcinoma and intestinal metaplasia, but not in normal gastric epithelium. Mild to strong TGR5 staining is associated with poor patient survival, and TCDA increased proliferation of a gastric adenocarcinoma cell line through the TGR5-dependent pathway (16). TDCA-induced ROS production and cell proliferation are mediated through TGR5 in Barrett's esophageal and esophageal adenocarcinoma cell lines (17). Stimulation with bile acids prompted the proliferation of an endometrial cell line by activating TGR5 and inducing cyclin D1 expression (18). In contrast to patients with gastric, esophageal and endometrial cancer, the binding of bile acids to TGR5 induced c-Jun-N terminal kinase (JNK) activation and enhanced apoptosis in hepatocytes (19). TGR5-deficient mice are much more susceptible to chemically induced acute liver injury with increased incidence of liver cancer (20). TGR5 may promote or suppress carcinogenesis after stimulation by bile acids (2). Since the role of bile acids in ampullary cancer is largely unknown, in the present study, we investigated the role of TGR5 in ampullary adenocarcinoma.

\section{Materials and methods}

Bioinformatic analysis. First, we conducted a search of the Kaplan-Meier plotter database (http://kmplot.com/analysis/) to systematically assess the expression level of the GPBAR1 gene in gastric, breast, lung and ovarian cancer patients (21-23). Kaplan-Meier Plot survival curves were drawn. Second, a PrognoScan database (http://www.abren.net/PrognoScan/) analysis was conducted. The expression level of the GPBARI gene was correlated with the survival of cancer patients. Third, data of GPBARI gene expression from genomics studies of 30 types of human cancer in the cBioPortal database (http://www.cbioportal.org/index.do) were examined $(24,25)$. Mutation of the GPBARl gene was documented by oncogenomic analysis.

Patients. A total of 99 patients who were diagnosed as having ampullary adenocarcinoma and who underwent radical resection at National Cheng Kung University Hospital from January 1990 to January 2010 were enrolled. Patients who received conservative treatment or exhibited other cell types of ampullary cancer were excluded. Demographics, histopathological findings and clinical outcomes were collected by conducting a retrospective chart review. A formal written informed consent was obtained from each patient. Their medical charts were reviewed until January 2016. The disease-specific survival rate was defined as the period from surgery until cancer-related death. The present study was approved by the Institutional Review Board of the National Cheng Kung University Hospital (NCKUH IRB no. A-ER-101-390 and B-ER-103-408).

Western blotting. Total protein lysates from tumor specimens and corresponding specimens of normal duodenum were obtained from the same patient and the protein concentration of the supernatants was measured using the amido black method. Equivalent amounts of protein $(30 \mu \mathrm{g})$ were separated on $10-15 \%$ polyacrylamide gels by SDS-gel electrophoresis, transferred to polyvinylidene difluoride membranes, and probed with the antibody against TGR5 (Abcam Biotechnology, Cambridge, UK), FXR (R\&D, Abingdon, UK), and GAPDH (Cell Signaling Technology, Danvers, MA, USA) proteins. Protein expression was visualized by ECL chemiluminescence (Promega, Madison, WI, USA) and quantitated by comparison with GAPDH.

Semi-quantitative reverse transcription polymerase chain reaction $(R T-P C R)$. The fresh cancer tissues and normal duodenum from the same patient, were obtained for RT-PCR. The total RNA was extracted from fresh tissues, and single-stranded cDNA was synthesized using oligo(dT) as the random primer. The cDNA was amplified using the primers for $\beta$-actin, GPBARl and $F X R$ genes, which were: $\beta$-actin sense, 5'-AGC GGG AAA TCG TGC GTG-3'; and $\beta$-actin antisense, 5'-CAG GGT ACA TGG TGG TGG TGC C-3'; GPBARl sense, 5'-CCC AGG CTA TCT TCC CAG C-3' and GPBARI antisense, 5'-GCC AGG ACT GAG AGG AGC A-3'; $F X R$ sense, 5'-GAC TTT GGA CCA TGA AGA CCA G-3' and $F X R$ antisense, 5'-GCC CAG ACG GAA GTT TCT TAT T-3'. The RT-PCR products were analyzed using agarose gel electrophoresis, and the GPBAR1 or FXR bands were semiquantified using densitometric analysis and subsequently normalized relative to the $\beta$-actin bands.

Immunohistochemical (IHC) staining. Samples of ampullary adenocarcinoma and the surrounding duodenum were fixed in $4 \%$ formalin and embedded in paraffin. IHC staining was performed using a monoclonal mouse anti-human TGR5 antibody (Abcam Biotechnology). The sections were incubated using an avidin-biotin complex reagent (Dako, Carpinteria, 
A

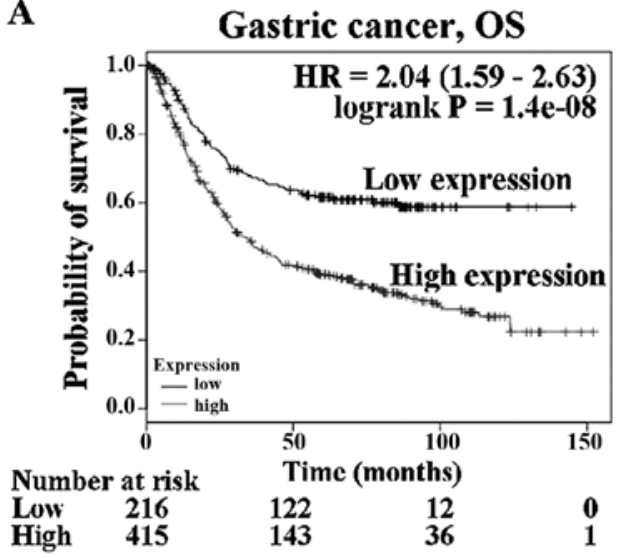

C

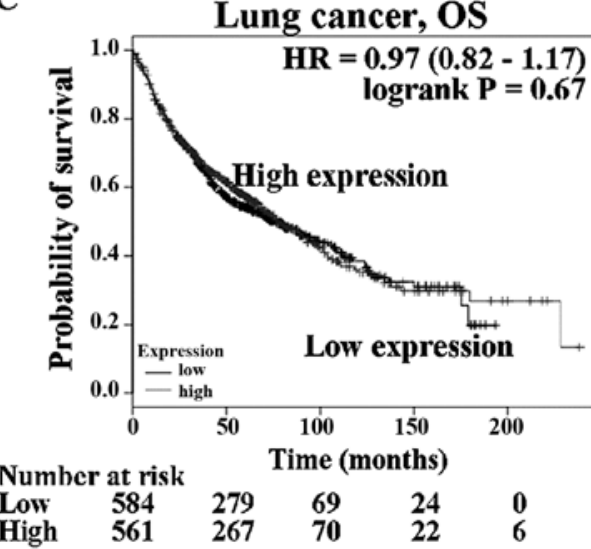

B

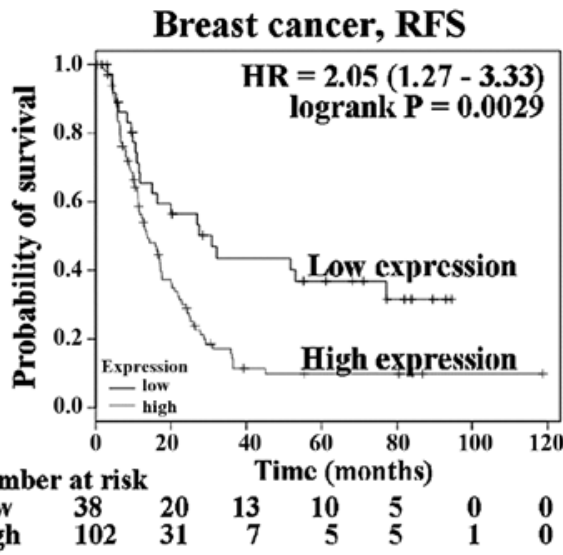

D

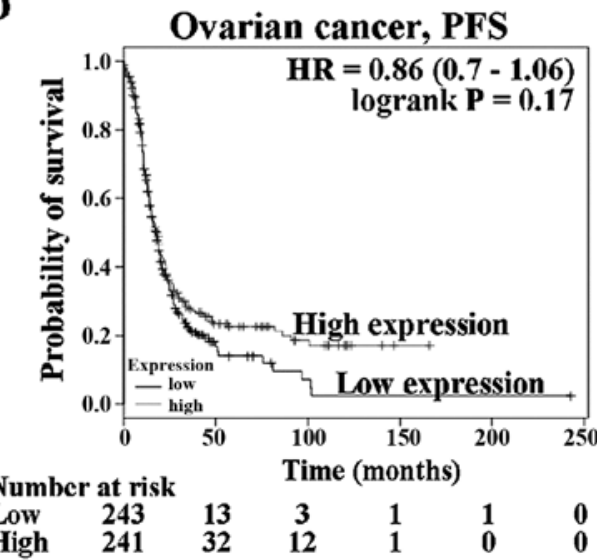

Figure 1. Kaplan-Meier survival analysis of GPBAR1 gene expression in cancer patients. The data were obtained from the Kaplan-Meier plotter database (http://kmplot.com/analysis/). High-GPBARl gene expression is indicated by the red line and low expression by the black line. (A) Overall survival of patients with gastric cancer. (B) Recurrence-free survival of patients with breast cancer. (C) Overall survival of patients with lung cancer. (D) Progression-free survival of patients with ovarian cancer. OS, overall survival; RFS, relapse-free survival; PFS, progression-free survival.

CA, USA), incubated with 3-amino-9-ethyl carbazole (Zymed Laboratories, South San Francisco, CA, USA) to develop the final color, and counterstained with hematoxylin. The immunoreactivity of the TGR5 protein was assessed using a semi-quantitative method and according to the Remmele and Stegner immunoreactive scoring (IRS) system (26). The IRS scores ranged from 0 to 12 and immunoreactivity was characterized as negative, weak, mild and strong. One researcher assessed the lesions (H.P. Hsu).

Statistical analysis. All statistical analyses were conducted using SPSS version 12.0 (SPSS, Inc., New York, NY, USA). A univariate analysis of the categorical variables was performed using the Chi-square test. The continuous variables were compared using the non-parametric Kruskal-Wallis $\mathrm{H}$ test. Any association between specific markers and the recurrence-free survival of patients was assessed using the Kaplan-Meier method, and the level of significance was tested using the log-rank test. A P-value of $<0.05$ was considered to indicate a statistically significant result.

\section{Results}

Analysis of microarray GAPBRl gene expression data. Several gene expression databases of human cancer genetics are available at websites, including the Kaplan-Meier plotter,
PrognoScan and cBioPortal system. There is no public database of ampullary adenocarcinoma genetics. Other human cancer types were used to study the function of TGR5 (gene name: GPBARl). These three databases were used to assess $G P B A R 1$ gene expression and correlate it with clinical outcome. Analysis of the relationship of the GPBARl gene expression level (based on Kaplan-Meier plotter data) to survival in patients with gastric, breast, lung and ovarian cancer (Fig. 1) revealed that prognosis was poorer in gastric cancer and breast cancer patients with high GPBARl gene expression than in those with low expression (Fig. 1A and B). GPBARl gene expression was not correlated with overall survival of patients with lung cancer (Fig. 1C). Ovarian cancer patients with high $G P B A R l$ gene expression tended to have a better prognosis than those with low expression (Fig. 1D). The function of GPBARl gene in tumor development differed between these four types of cancer.

Prognoscan is a collection of human cancer microarray datasets. High GPBARl gene expression predicted a trend toward poor prognosis in 12 datasets (Table I) and good prognosis in 15 datasets (Table II). In most datasets, the GPBARl gene expression was not significantly correlated with survival and only one dataset in each group displayed predictive power (GSE13507 and GSE8894). The level of GPBAR1 expression in human cancer was investigated using the cBioPortal system 


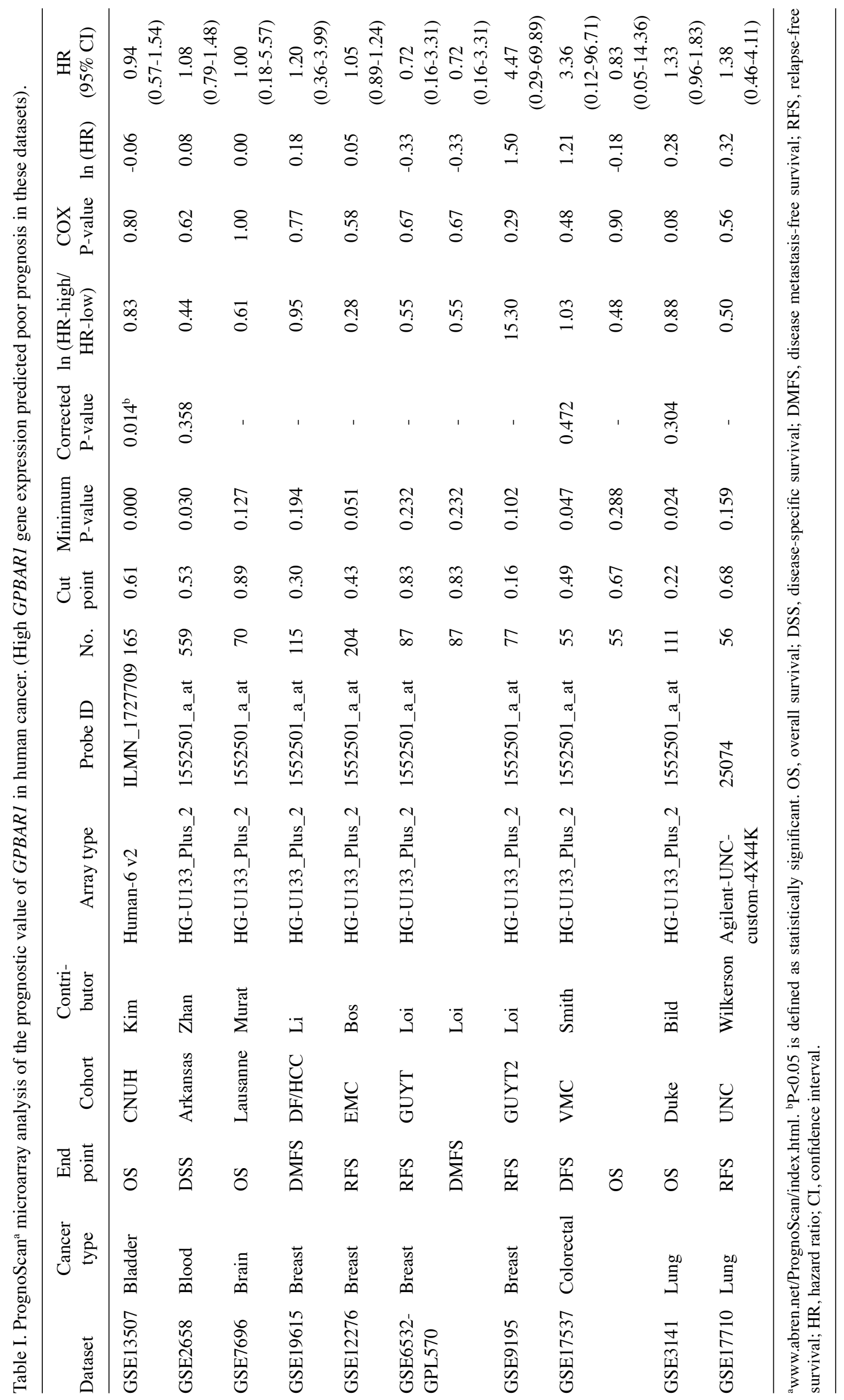




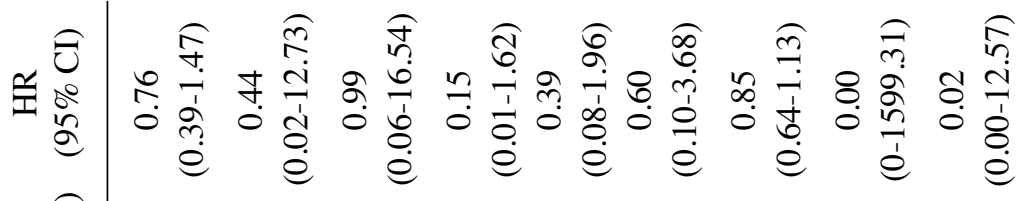

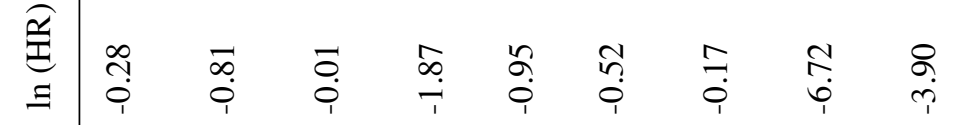

希

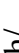

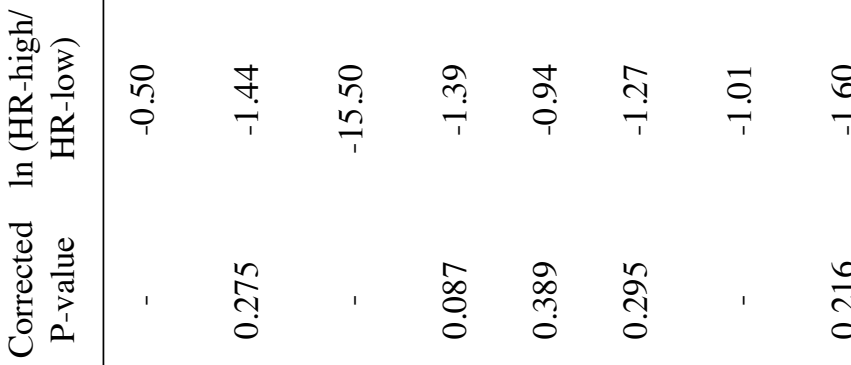

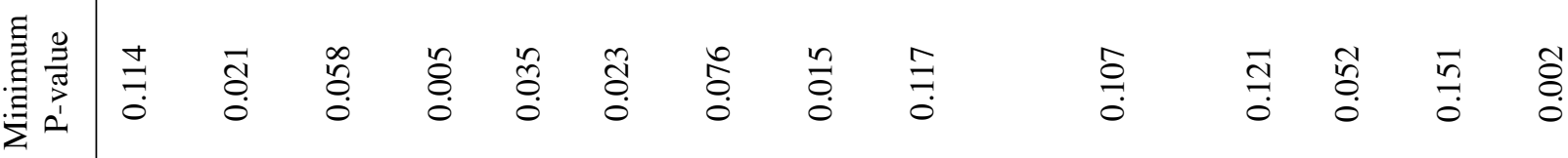

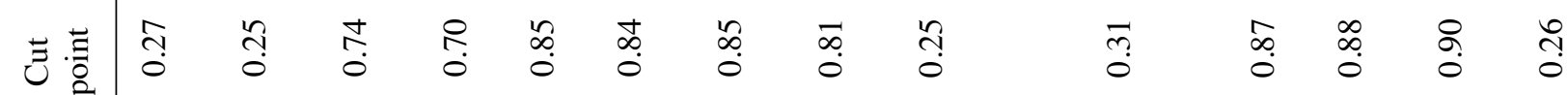

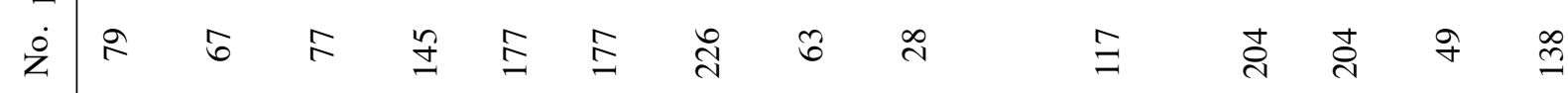

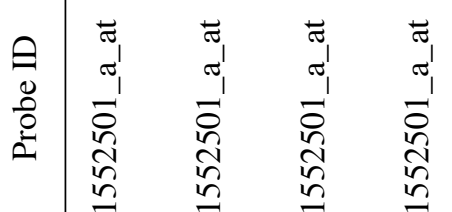

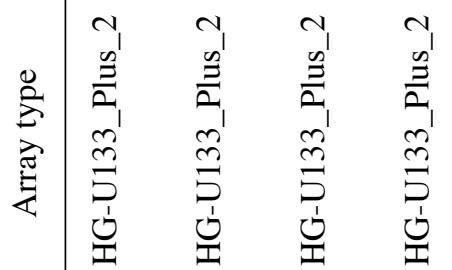

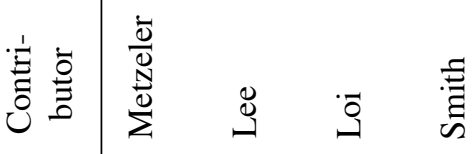

蓠

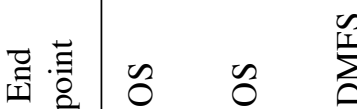

芯

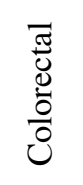

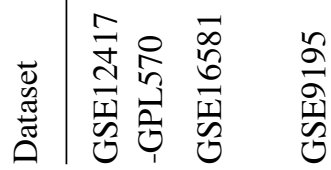

$\frac{\infty}{n}$

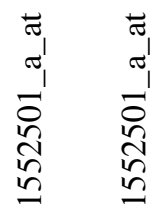

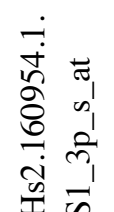

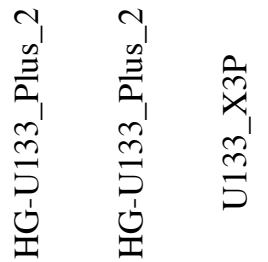

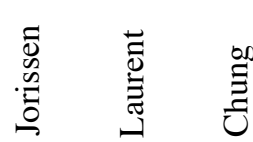

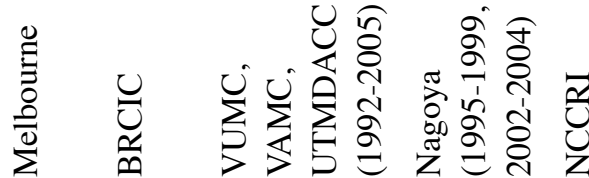

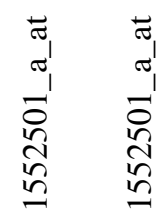

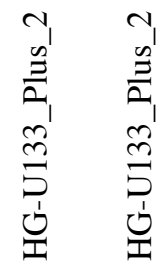

莺

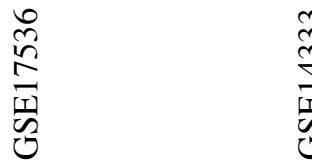

要

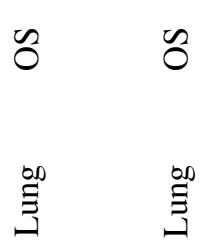

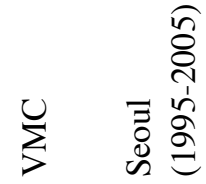

离

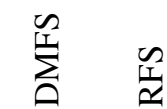

్ㅡ

爷莺苞

$\frac{m}{\sqrt{2}}$ $\frac{0}{3}$

毫歖 


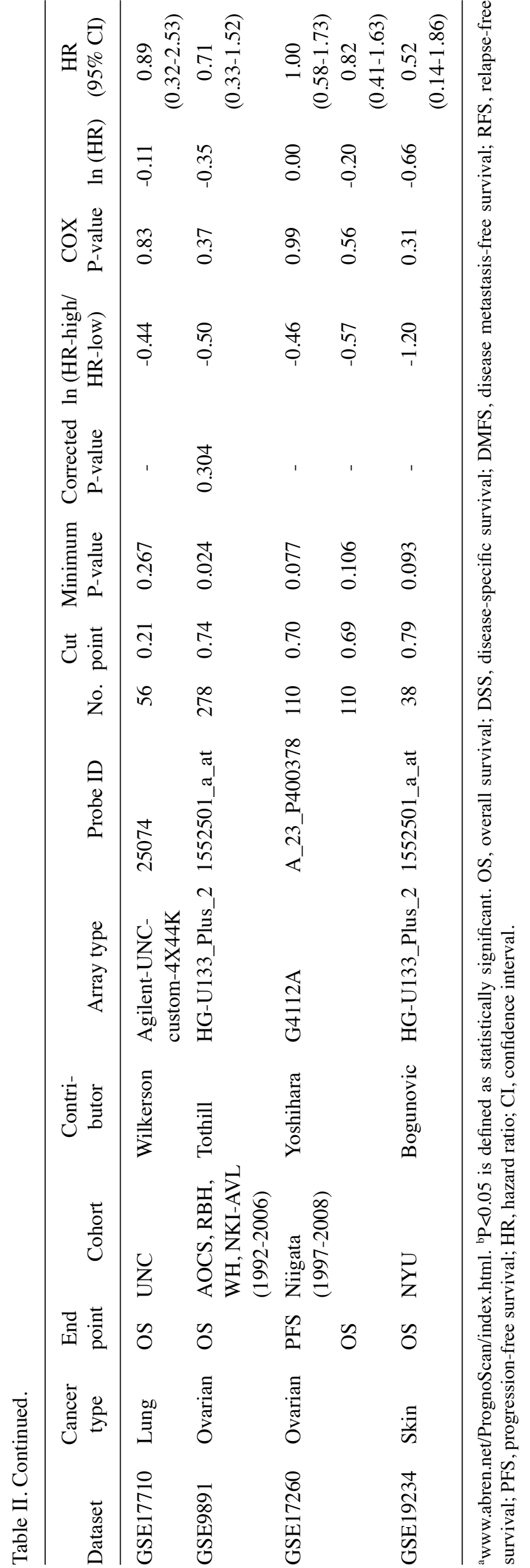

and analyzed in 30 types of cancer (Fig. 2A). Three specific types of cancer (cholangiocarcinoma, pancreatic and colorectal cancer) were compared (Fig. 2B). Cholangiocarcinoma and pancreatic cancer are continuously exposed to bile and toxic bile acids are well-established carcinogens for cholangiocarcinoma and colorectal cancer. Cholangiocarcinoma and pancreatic cancer, compared to other cancers, had a higher level of GPBARI expression (Fig. 2B). Thus the function of $G P B A R 1$ is distinct in different cancers, and its expression may be correlated with bile exposure.

Expression of TGR5 protein in ampullary cancer. The ampulla of Vater is located in the second part of the duodenum and is exposed to bile acids under normal physiological conditions. TGR5 protein (product of the GPBARI gene) and GPBARI mRNA were detected in clinical samples of ampullary cancer and the surrounding normal duodenum (Fig. 3). In the patients with cancer recurrence, the TGR5 protein level was lower in the tumor than that noted in the normal duodenum. In the patients without cancer recurrence, the tumor TGR5 level was similar to the normal tissue level. In contrast, no such pattern was found for FXR protein (Fig. 3A). Increased GPBARI mRNA was detected in 7 of 15 specimens of ampullary cancer, particularly in well-differentiated ampullary adenocarcinoma (Fig. 3B and C). Expression of FXR mRNA was not correlated with histological differentiation (Fig. 3C).

Immunohistochemical staining of TGR5 in ampullary cancer. To study the relationship of TGR5 protein expression with clinical outcome, 99 specimens of ampullary adenocarcinoma were immunostained for TGR5. TGR5 was detected in the cytoplasm and nucleus of each cancer cell (Fig. 4). We divided the result as negative, weak, mild and strong expression of TGR5. Expression of TGR5 was negative in 14 patients, weak in 33, mild in 29, and strong in 23 (Table I). Negative, weak or mild expression of TGR5 was correlated with younger age $(\mathrm{P}=0.043)$ and higher level of direct bilirubin $(\mathrm{P}=0.023$, separately) and tended to be correlated with higher level of total bilirubin ( $\mathrm{P}=0.059)$, higher plasma level of cancer antigen-125 (CA-125) ( $\mathrm{P}=0.099)$, advanced tumor stage and AJCC TNM stage $(\mathrm{P}=0.063$ and 0.062 , separately) (Table III).

Correlation of TGR5 expression with clinical outcomes of ampullary cancer patients. In 95 patients with regular follow-up (range, 3-249 months), 55 patients developed recurrence. The recurrences in patients with negative, weak or mild TGR5 expression tended to be earlier (within postoperative 12 months) $(\mathrm{P}=0.089)$, although the level of TGR5 expression was not associated with recurrence patterns (Table IV). The disease-specific survival rate tended to be better in patients with strong TGR5 expression ( $\mathrm{P}=0.1118$; Fig. 5A).

In the literature, the function of TGR5 depends on dysregulation of bile acid homeostasis (2). We hypothesized that high levels of preoperative bilirubin interacts with TGR5 in ampullary cancer. We grouped the patients according to the median level of total bilirubin $(2.45 \mathrm{mg} / \mathrm{dl})$. Although not correlated with survival in the patients with total bilirubin $\leq 2.45 \mathrm{mg} / \mathrm{dl}$ (Fig. 5B), TGR5 expression predicted a better prognosis in patients with higher than $2.45 \mathrm{mg} / \mathrm{dl}(\mathrm{P}=0.0464$; Fig. 5C). 

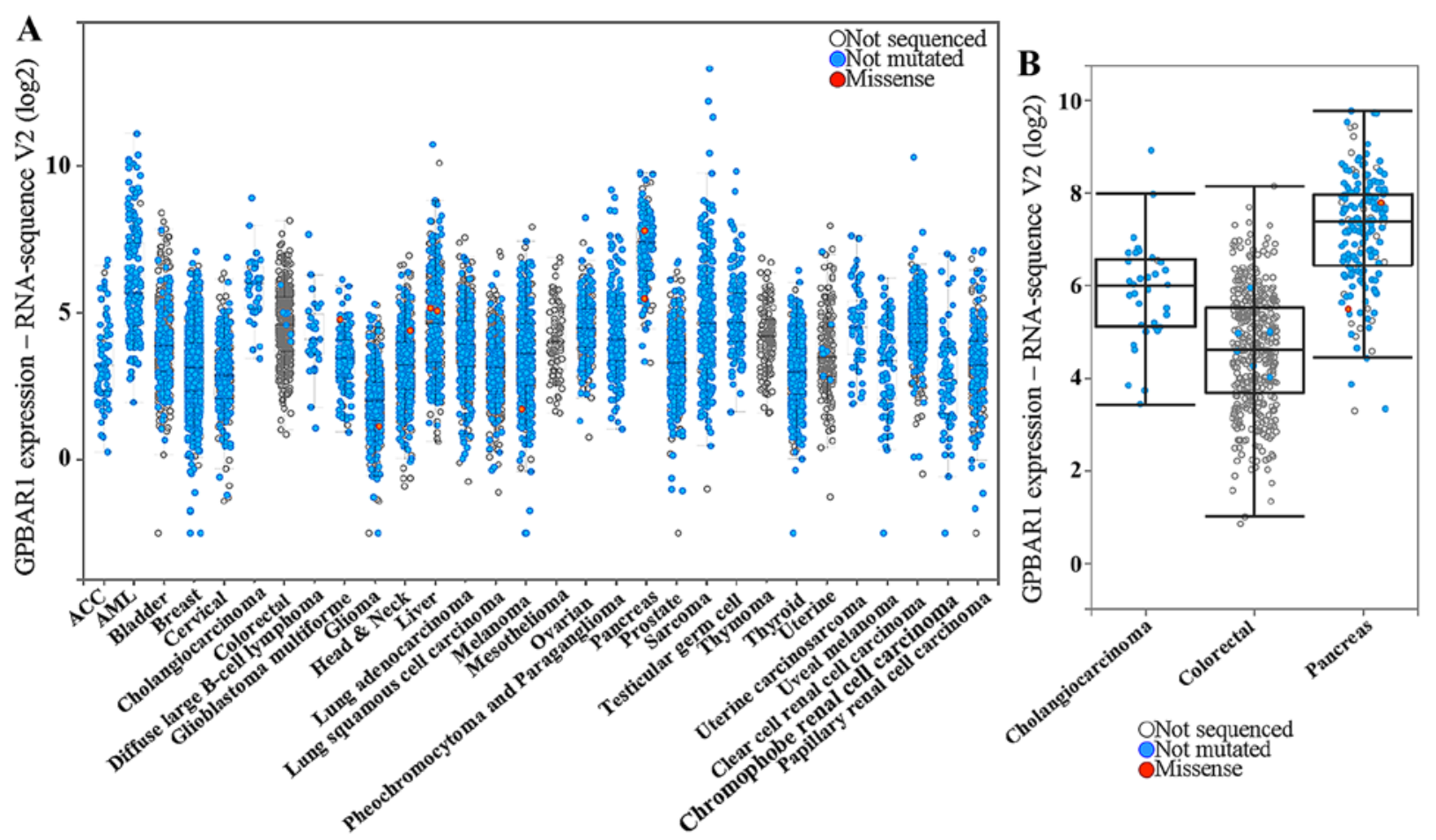

Figure 2. Analysis of GPBARI expression level data from the cBioPortal database (http://www.cbioportal.org/index.do). Every spot represents a single study. White spots represent those analyzed without gene sequencing, blue spots represent normal results of gene sequencing and red spots represent missense mutations. (A) Level of GPBARI expression in 30 types of human cancer. (B) Level of GPBAR1 expression in three specific-types of cancer (bile acid-exposed cholangiocarcinoma and pancreatic cancer vs. colorectal cancer). The median and interquartile range are presented. The median level of GPBARl gene expression was higher in cholangiocarcinoma and pancreatic cancer than in colorectal cancer.
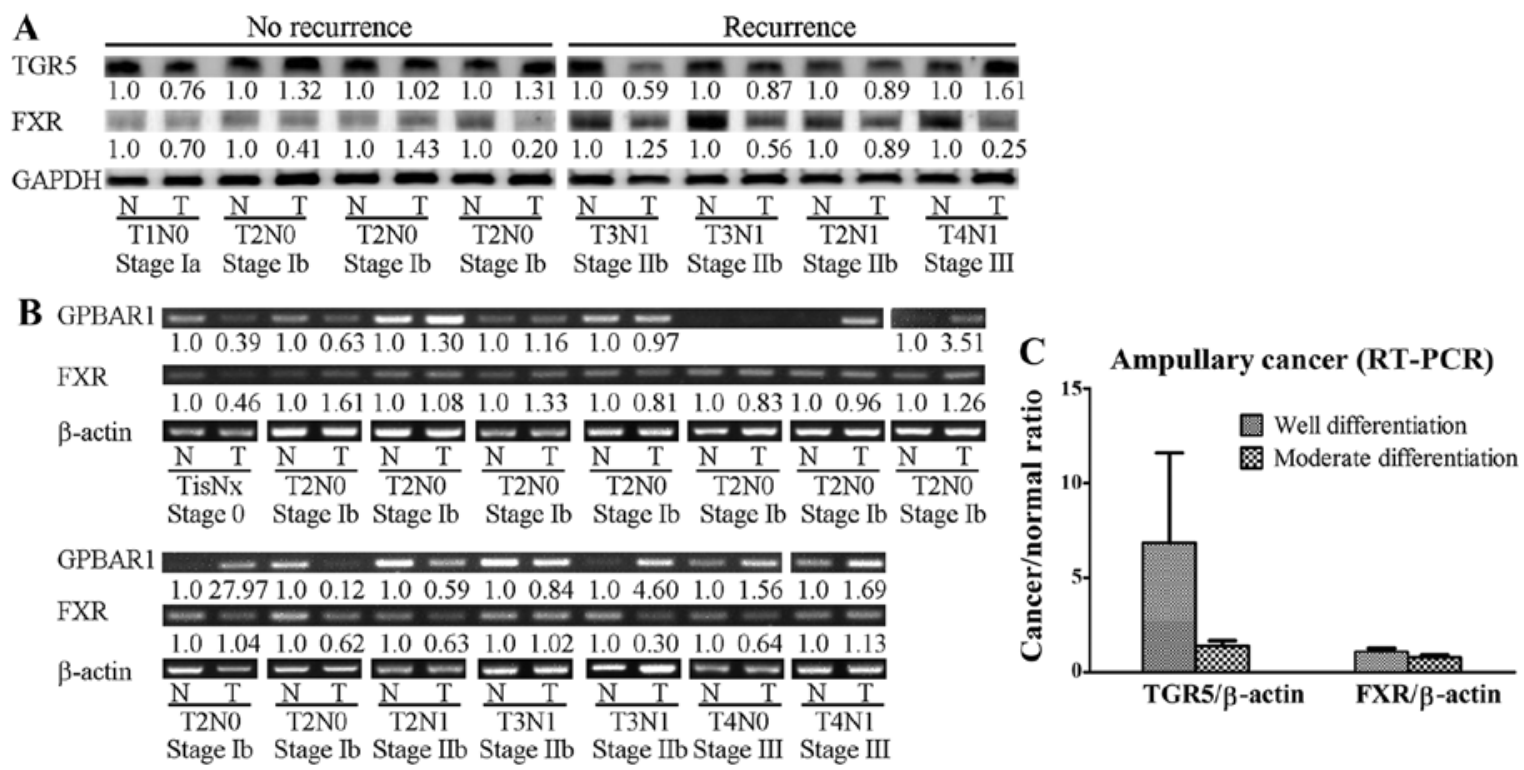

Figure 3. Expression of TGR5 and FXR in ampullary adenocarcinoma. Sample pairs consisting of ampullary adenocarcinoma (T) and its corresponding normal duodenum $(\mathrm{N})$ were collected. (A) Eight sample pairs were assessed by western blotting of TGR5 and FXR proteins with GAPDH serving as a loading control. The fold-change of TGR5/GAPDH or FXR/GAPDH is indicated below the band. (B) A total of 15 sample pairs were assessed by semi-quantitative RT-PCR, with $\beta$-actin serving as a loading control. The fold-change of TGR5/ $\beta$-actin or FXR/ $\beta$-actin is indicated below the band. (C) The proportion of TGR5/ $\beta$-actin or FXR/ $\beta$-actin expressed in tumors relative to normal control tissues was correlated with histological differentiation.

\section{Discussion}

The ampullary of Vater is normally exposed to bile. This is the first study to investigate expression of the membrane bile acid receptor, TGR5, in ampullary adenocarcinoma. The results of analysis of GPBAR1 gene expression data (gene of TGR5) in microarray databases was correlated with clinical outcomes and varied between types of cancers. The pathological function of TGR5 in cancer is regulated by a complex mechanism. In ampullary adenocarcinoma, the present study detected TGR5 
Table III. Correlation of TGR5 expression with demographics and histopathological findings in patients with ampullary adenocarcinoma who underwent radical resection.

\begin{tabular}{|c|c|c|c|}
\hline & \multicolumn{2}{|c|}{ Expression of TGR5 } & \multirow[b]{2}{*}{ P-value } \\
\hline & Negative, weak, mild & Strong & \\
\hline Patients, n (\%) & $76(77)$ & $23(23)$ & \\
\hline Gender, n (\%) & & & 0.481 \\
\hline Female & $30(73)$ & $11(27)$ & \\
\hline Male & $46(79)$ & $12(21)$ & \\
\hline Age at surgery (years) ${ }^{a}$ & $65(32-90)$ & $68(35-83)$ & 0.043 \\
\hline Total bilirubin $(\mathrm{mg} / \mathrm{dl})^{\mathrm{a}}$ & $3.4(0.2-19.6)$ & $1.3(0.4-16.3)$ & 0.059 \\
\hline Direct bilirubin $(\mathrm{mg} / \mathrm{dl})^{\mathrm{a}}$ & $2.6(0-18.0)$ & $0.6(0-7.3)$ & 0.023 \\
\hline Preoperative bile decompression, n (\%) & $41(77)$ & $12(23 \%)$ & 1.000 \\
\hline $\mathrm{CEA}(\mathrm{ng} / \mathrm{ml})^{\mathrm{a}}$ & $1.9(0.1-296.3)$ & $2.5(0-13.0)$ & 0.306 \\
\hline CA-125 (U/ml $)^{\mathrm{a}}$ & $15.4(5.2-164.1)$ & $11.5(0.5-66.7)$ & 0.099 \\
\hline CA-199 (U/ml $)^{\mathrm{a}}$ & $55.4(0.3-7512.9)$ & $44.2(1.4-1860)$ & 0.892 \\
\hline Subtype, $\mathrm{n}(\%)^{\mathrm{b}}$ & & & 0.206 \\
\hline Intestinal type & $37(74)$ & $13(26)$ & \\
\hline Pancreaticoduodenal type & $17(90)$ & $2(10)$ & \\
\hline Tumor type, $\mathrm{n}(\%)$ & & & 0.315 \\
\hline Polypoid & $40(74)$ & $14(26)$ & \\
\hline Ulcerative & $21(87)$ & $3(13)$ & \\
\hline Mixed & $15(71)$ & $6(29)$ & \\
\hline Resection margin, $\mathrm{n}(\%)$ & & & 1.000 \\
\hline Free & $66(76)$ & $21(24)$ & \\
\hline Microscopically positive & $8(80)$ & $2(20)$ & \\
\hline Lymph node metastasis, $\mathrm{n}(\%)^{\mathrm{b}}$ & & & 0.176 \\
\hline Negative & $42(75)$ & $14(25)$ & \\
\hline Positive & $30(88)$ & $4(12)$ & \\
\hline Lymphovascular invasion, $\mathrm{n}(\%)^{\mathrm{b}}$ & & & 0.192 \\
\hline Negative & $27(69)$ & $12(31)$ & \\
\hline Positive & $34(83)$ & $7(17)$ & \\
\hline Perineural invasion, $\mathrm{n}(\%)^{\mathrm{b}}$ & & & 0.373 \\
\hline Negative & $28(68)$ & $13(32)$ & \\
\hline Positive & $18(82)$ & $4(18)$ & \\
\hline Histological differentiation, $\mathrm{n}(\%)^{\mathrm{b}}$ & & & 0.847 \\
\hline Well & $31(74)$ & $11(26)$ & \\
\hline Moderate & $37(77)$ & $11(23)$ & \\
\hline Poor & $5(83)$ & $1(17)$ & \\
\hline Pancreatic invasion, $\mathrm{n}(\%)^{\mathrm{b}}$ & & & 0.149 \\
\hline Negative & $34(71)$ & $14(29)$ & \\
\hline Positive & $42(84)$ & $8(16)$ & \\
\hline Tumor size $(\mathrm{cm})^{\mathrm{a}}$ & $2.4(0.7-8.0)$ & $2.5(1.0-6.0)$ & 0.783 \\
\hline Tumor stage, n (\%) & & & 0.063 \\
\hline $\mathrm{T} 1$ & $5(45)$ & $6(55)$ & \\
\hline $\mathrm{T} 2$ & $27(75)$ & $9(25)$ & \\
\hline $\mathrm{T} 3$ & $29(83)$ & $6(17)$ & \\
\hline $\mathrm{T} 4$ & $15(88)$ & $2(12)$ & \\
\hline AJCC TNM stage, $\mathrm{n}(\%)$ & & & 0.062 \\
\hline I & $26(63)$ & $15(37)$ & \\
\hline II & $34(85)$ & $6(15)$ & \\
\hline III & $15(88)$ & $2(12)$ & \\
\hline IV & $1(100)$ & $0(0)$ & \\
\hline
\end{tabular}

${ }^{a}$ Values are expressed as median (range). ${ }^{b}$ Excluding patients without detailed records. AJCC TNM stage, American Joint Committee on Cancer tumor-node-metastases (TNM) staging system. 

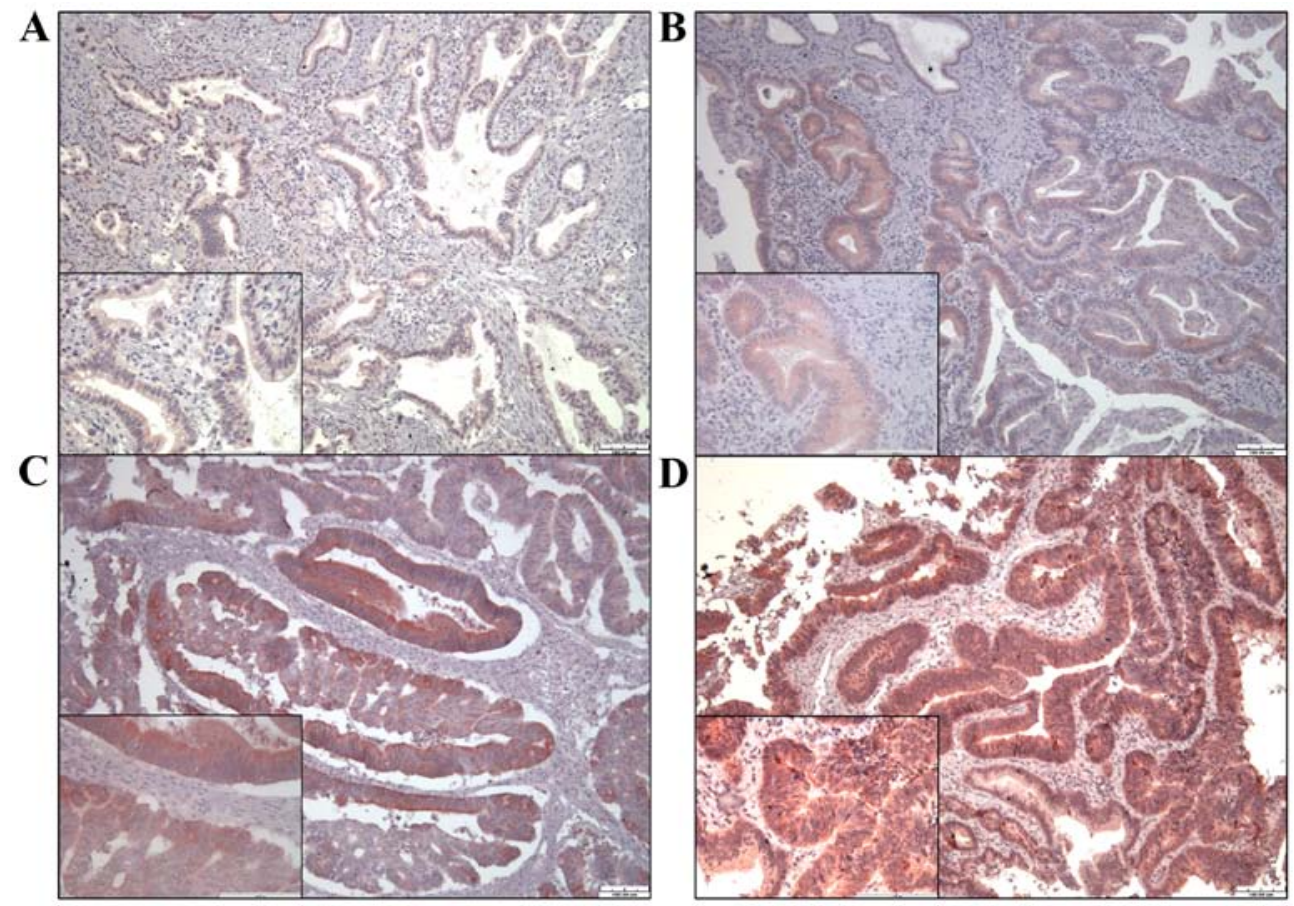

Figure 4. Expression of TGR5 in ampullary adenocarcinoma was assessed by immunohistochemical (IHC) staining and rated using the Remmele and Stegner immunoreactive scoring system. Expression was either (A) 0-1 (negative); (B) 2-3 (weak); (C) 4-8 (mild) or (D) 9-12 (strong).

Table IV. Correlation between disease recurrence and TGR5 expression in patients with ampullary adenocarcinoma who underwent radical resection.

\begin{tabular}{lccr}
\hline & \multicolumn{2}{c}{ Expression of TGR5 } & P-value \\
\cline { 2 - 3 } & Negative, weak, mild & Strong & $23(23)$ \\
\hline Patients, $\mathrm{n}(\%)$ & $76(77)$ & $12(30)$ & \\
No recurrence, $\mathrm{n}(\%)^{\mathrm{a}}$ & $28(70)$ & $11(20)$ & 0.089 \\
Recurrence, $\mathrm{n}(\%)^{\mathrm{a}}$ & $44(80)$ & & \\
Delayed recurrence, $\mathrm{n}(\%)$ & & & \\
$\quad($ after postoperative 12 months) & $16(70)$ & $30)$ & 0.260 \\
Early recurrence, $\mathrm{n}(\%)$ & $28(90)$ & $3(10)$ & 0.205 \\
$\quad($ within postoperative 12 months) & $19(86)$ & $3(14)$ & 0.506 \\
Patterns of recurrence, $\mathrm{n}(\%)^{\mathrm{a}, \mathrm{b}}$ & $28(85)$ & $5(15)$ & 0.437 \\
Liver metastasis, $\mathrm{n}(\%)$ & $12(86)$ & $2(14)$ & 0.754 \\
Local recurrence, $\mathrm{n}(\%)$ & $6(67)$ & $3(33)$ & $3(18)$ \\
Peritoneal carcinomatosis, $\mathrm{n}(\%)$ & $14(82)$ & & \\
Bone metastasis, $\mathrm{n}(\%)$ & & & \\
Other metastasis, $\mathrm{n}(\%)^{\mathrm{c}}$ & & & \\
\hline
\end{tabular}

${ }^{\mathrm{a}}$ Excludes two patients who died due to surgical complications and two patients who were lost in the follow-up in our hospital. ${ }^{\mathrm{b}}$ Some patients developed more than one type of metastases. 'Including brain, lung and ovary metastases.

protein and GPBARI mRNA in the tumor and surrounding normal duodenum. Negative, weak or mild TGR5 expression was correlated with elevation of plasma bilirubin. In the patients with plasma total bilirubin higher than the median, strong TGR5 expression predicted a better prognosis.

There are two types of ampullary adenocarcinoma: intestinal and pancreaticobiliary types. These differ in clinical behavior (27) and the differences may be intrinsic. Nuclear accumulation of $\beta$-catenin promotes WNT activation and cancer progression; however, loss of the $\beta$-catenin protein in ampullary cancer is correlated with poor prognosis (28). Nestin, a stemness protein, performs a dual role in ampullary adenocarcinoma, as a predictor of good prognosis in early cancer and as a promoter of metastasis in advanced 
A

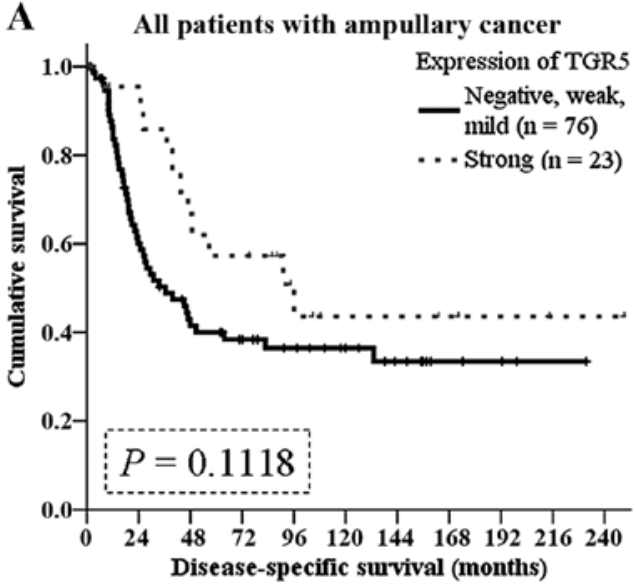

B
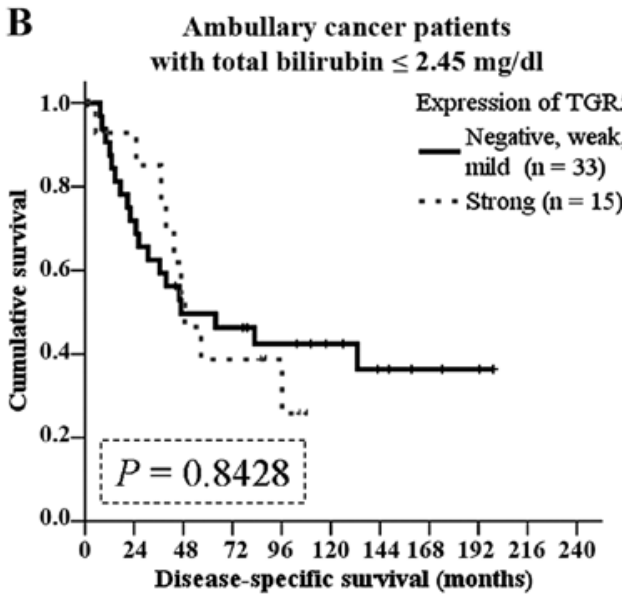

C

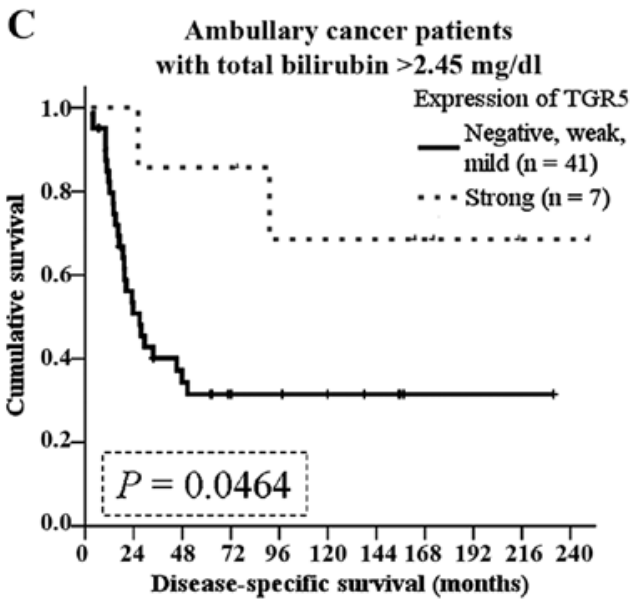

Figure 5. Kaplan-Meier analysis of the impact of TGR5 expression on disease-specific survival in patients with ampullary adenocarcinoma. (A) Disease-specific survival of all patients with ampullary adenocarcinoma who underwent surgery according to TGR5 expression level $(\mathrm{P}=0.1118)$ (B) Disease-specific survival of ampullary cancer patients with plasma total bilirubin concentration $\leq 2.45 \mathrm{mg} / \mathrm{dl}$. TGR5 expression level was not correlated with survival $(\mathrm{P}=0.8428)$. (C) Disease-specific survival of ampullary cancer patients with plasma total bilirubin concentration $>2.45 \mathrm{mg} / \mathrm{dl}$. Strong TGR5 expression predicted a better survival $(\mathrm{P}=0.0464)$.

cancer (29). The epithelial cell marker, EpCAM, is one of the signatures of cancer stem cells with oncogenic potential mediated via upregulation of c-myc and cyclins. However, loss of EpCAM is linked to a more aggressive phenotype of ampullary cancer, suggesting that EpCAM may play a different role in ampullary cancer than in other cancers (30). Ampullary adenocarcinoma is a unique cancer with a 5-year survival rate $<50 \%$ after curative resection $(27,31,32)$. Further study of ampullary adenocarcinoma is required to develop new treatment modalities and improve clinical outcomes.

The ampullary of Vater is located at the confluence of the common bile and pancreatic ducts, and second portion of the duodenum. Long-term exposure to bile acids increases oxidative stress, generates ROS/RNS, and induces cell damage and mutation rates in gastrointestinal cancer $(2,5,6)$. Alteration of the bile contents of gastroesophageal reflux is correlated with the increased incidence of cancer in cell culture, animal models and epidemiology studies $(3,4,33)$. Toxic bile acids induce expression of COX-2 or activation of EGFR and promote carcinogenesis in cholangiocarcinoma $(7,8)$. In our previous study, preoperative plasma concentration of total bilirubin was elevated in non-survivors of ampullary cancer (32). However, no relationship was found between hyperbilirubinemia and ampullary cancer recurrence.

Bile acids interact not only with nuclear receptors, but also with membrane receptors. TGR5 is a G protein-coupled bile acid receptor that mediates bile acid-regulated energy and glucose homeostasis $(2,9,34)$. Bile acids induce cell proliferation and cell cycle progression through the TGR5-dependent pathway and TGR5 acts such as an oncoprotein (16-18). In hepatocytes, suppression of TGR5 enhances chemicalinduced carcinogenesis and activation of TGR5 promotes cell apoptosis $(19,20)$. Whether TGR5 promotes or suppresses carcinogenesis depends on the composition of the bile acids (2). We analyzed multiple microarray datasets and found that high GPBARl gene expression predicted poor prognosis in some datasets, but good prognosis in others (Tables I and II; Fig. 1). Bile acid-exposed cancers (such as cholangiocarcinoma and pancreatic cancer) had a higher ratio of GPBARl expression (Fig. 2B). Since the ampulla of Vater is also exposed to bile acids under normal physiological conditions, the study of bile acid receptors, such as TGR5 and FXR, is indicated in ampullary cancer. In the present study, the mRNAs or proteins of TGR5 or FXR were detected in specimens of ampullary adenocarcinoma (Fig. 3). Increased expression of GPBARl mRNA but not FXR mRNA was correlated with histological differentiation and well-differentiated adenocarcinoma (Fig. 3C). Taken together, our results indicate that the membrane receptor of bile acids, TGR5, may be activated in ampullary cancer.

Activation of TGR5 plays a role in cyclic adenosine monophosphate (cAMP), EGFR, mitogen-activated protein kinase (MAPK, such as JNK, ERK-1/2), cyclooxygenase-2 (COX-2) or signal transducer and activator of transcription 3 (STAT3) signaling (35). TGR5 functions in a cell type-dependent and context-dependent manner in cancer. In gastric and esophageal cancer, the TGR5-dependent pathway mediates bile acid-induced ROS production and cell proliferation as well as deoxycholate-induced EGFR phosphorylation and ERK1/2 activation. Moreover, TGR5 expression is associated with the poor prognosis of patients (36-38), suppresses STAT3 signaling and inhibits cell cycle progression, angiogenesis, metastasis and evasion of the immune system in gastric cancer (36). Interaction of TGR5 and EGFR depends on lipid rafts. Deoxycholate induces EGFR phosphorylation and ERK1/2 activation through the TGR5-dependent pathway (38). However, TGR5 performs 
as a tumor-suppressor in liver cancer. TGR5-deficient mice have an increased incidence of liver cancer (19). Bile acids conjugate TGR5 to induce JNK activation and enhance apoptosis in hepatocytes (20). In the present study, strong TGR5 expression was correlated with lower plasma concentration of total and direct bilirubin. The patients with strong TGR5 expression tended to have a lower plasma level of CA-125, earlier tumor stage, and earlier AJCC TNM stage and also a better disease-specific survival rate, particularly those patients with total bilirubin concentration higher than $2.45 \mathrm{mg} / \mathrm{dl}$. We conclude that TGR5 performs as a tumor suppressor in hyperbilirubinemic patients with ampullary adenocarcinoma.

In summary, high TGR5 expression was correlated with lower plasma concentration of total/direct bilirubin, lower plasma level of CA-125, early tumor stage and AJCC TNM stage. High TGR5 expression also predicted a good survival in patients with total bilirubin levelss higher than $2.45 \mathrm{mg} / \mathrm{dl}$. TGR5 performs as a tumor suppressor in hyperbilirubinemia condition of ampullary adenocarcinoma patients.

\section{Acknowledgements}

The present study was supported by grants from the National Science Council (grant NSC-102-B-2314-B-006-043), the Ministry of Science and Technology (grant MOST-104-B2314-B-006-049), the National Cheng Kung University Hospital (to H.-P.H.), and the Chi Mei Medical Center (to M.-C.C.). The authors are grateful for the support from the Human Biobank, Research Center of Clinical Medicine, National Cheng Kung University Hospital. We were blessed with support from the late superintendent, Professor Pin-Wen Lin. Furthermore, we thank Mr. Chih-Yang Wang and Ms. Yu-Hsuan Hung for their support.

\section{References}

1. Albores-Saavedra J, Schwartz AM, Batich K and Henson DE: Cancers of the ampulla of vater: Demographics, morphology, and survival based on 5,625 cases from the SEER program. J Surg Oncol 100: 598-605, 2009.

2. Tsuei J, Chau T, Mills D and Wan YJ: Bile acid dysregulation, gut dysbiosis, and gastrointestinal cancer. Exp Biol Med 239: $1489-1504,2014$

3. Ye W, Chow WH, Lagergren J, Yin L and Nyrén O: Risk of adenocarcinomas of the esophagus and gastric cardia in patients with gastroesophageal reflux diseases and after antireflux surgery. Gastroenterology 121: 1286-1293, 2001.

4. Nishioka K, Doki Y, Miyata H, Tamura S, Yasuda T, Kimura Y, Kishi K, Yoshida K, Fujiwara Y, Yano M, et al: Bile acid promotes the proliferation of squamous cell carcinoma of the esophagus, independent of its inducing COX-2 expression. J Surg Res 132: 130-135, 2006.

5 Bernstein C, Payne CM and Bernstein H: Bile acids: Promoters or carcinogens in colon cancer? J Carcinogene Mutagene 2: 101e. doi:10.4172/2157-2518.1000101e, 2011.

6. Payne CM, Bernstein C, Dvorak K and Bernstein H: Hydrophobic bile acids, genomic instability, Darwinian selection, and colon carcinogenesis. Clin Exp Gastroenterol 1: 19-47, 2008.

7. Komichi D, Tazuma S, Nishioka T, Hyogo H and Chayama K: Glycochenodeoxycholate plays a carcinogenic role in immortalized mouse cholangiocytes via oxidative DNA damage. Free Radic Biol Med 39: 1418-1427, 2005.

8. Yoon JH, Higuchi H, Werneburg NW, Kaufmann SH and Gores GJ: Bile acids induce cyclooxygenase- 2 expression via the epidermal growth factor receptor in a human cholangiocarcinoma cell line. Gastroenterology 122: 985-993, 2002.

9. Thomas C, Pellicciari R, Pruzanski M, Auwerx J and Schoonjans K: Targeting bile-acid signalling for metabolic diseases. Nat Rev Drug Discov 7: 678-693, 2008.
10. Lax S, Schauer G, Prein K, Kapitan M, Silbert D, Berghold A Berger A and Trauner M: Expression of the nuclear bile acid receptor/farnesoid $\mathrm{X}$ receptor is reduced in human colon carcinoma compared to nonneoplastic mucosa independent from site and may be associated with adverse prognosis. Int J Cancer 130: 2232-2239, 2012

11. van de Winkel A, van Zoest KP, van Dekken H, Moons LM, Kuipers EJ and van der Laan LJ: Differential expression of the nuclear receptors farnesoid $\mathrm{X}$ receptor $(\mathrm{FXR})$ and pregnane $\mathrm{X}$ receptor (PXR) for grading dysplasia in patients with Barrett's oesophagus. Histopathology 58: 246-253, 2011.

12. Kim I, Morimura K, Shah Y, Yang Q, Ward JM and Gonzalez FJ: Spontaneous hepatocarcinogenesis in farnesoid $\mathrm{X}$ receptor-null mice. Carcinogenesis 28: 940-946, 2007.

13. Li T, Holmstrom SR, Kir S, Umetani M, Schmidt DR, Kliewer SA and Mangelsdorf DJ: The G protein-coupled bile acid receptor, TGR5, stimulates gallbladder filling. Mol Endocrinol 25: 1066-1071, 2011

14. Thomas C, Gioiello A, Noriega L, Strehle A, Oury J, Rizzo G, Macchiarulo A, Yamamoto $\mathrm{H}$, Mataki C, Pruzanski M, et al: TGR5-mediated bile acid sensing controls glucose homeostasis. Cell Metab 10: 167-177, 2009.

15. Duboc H, Taché Y and Hofmann AF: The bile acid TGR5 membrane receptor: From basic research to clinical application. Dig Liver Dis 46: 302-312, 2014.

16. Cao W, Tian W, Hong J, Li D, Tavares R, Noble L, Moss SF and Resnick MB: Expression of bile acid receptor TGR5 in gastric adenocarcinoma. Am J Physiol Gastrointest Liver Physiol 304: G322-G327, 2013.

17. Hong J, Behar J, Wands J, Resnick M, Wang LJ, DeLellis RA, Lambeth D, Souza RF, Spechler SJ and Cao W: Role of a novel bile acid receptor TGR5 in the development of oesophageal adenocarcinoma. Gut 59: 170-180, 2010.

18. Casaburi I, Avena P, Lanzino M, Sisci D, Giordano F, Maris P, Catalano S, Morelli $\mathrm{C}$ and Andò S: Chenodeoxycholic acid through a TGR5-dependent CREB signaling activation enhances cyclin D1 expression and promotes human endometrial cancer cell proliferation. Cell Cycle 11: 2699-2710, 2012.

19. Yang JI, Yoon JH, Myung SJ, Gwak GY, Kim W, Chung GE, Lee SH, Lee SM, Kim CY and Lee HS: Bile acid-induced TGR5-dependent c-Jun-N terminal kinase activation leads to enhanced caspase 8 activation in hepatocytes. Biochem Biophys Res Commun 361: 156-161, 2007.

20. Chen WD, Yu D, Forman BM, Huang W and Wang YD: Deficiency of G-protein-coupled bile acid receptor Gpbar1 (TGR5) enhances chemically induced liver carcinogenesis. Hepatology 57: 656-666, 2013.

21. Györffy B, Lánczky A, Eklund AC, Denkert C, Budczies J, Li Q and Szallasi Z: An online survival analysis tool to rapidly assess the effect of 22,277 genes on breast cancer prognosis using microarray data of 1,809 patients. Breast Cancer Res Treat 123: 725-731, 2010.

22. Győrffy B, Surowiak P, Budczies J and Lanczky A: Online survival analysis software to assess the prognostic value of biomarkers using transcriptomic data in non-small-cell lung cancer. PLoS One 8: e82241.1-e82241.8, 2013.

23. Győrffy B, Lánczky A and Szállási Z: Implementing an online tool for genome-wide validation of survival-associated biomarkers in ovarian-cancer using microarray data from 1287 patients. Endocr Relat Cancer 19: 197-208, 2012.

24. Gao J, A ksoy BA, Dogrusoz U, Dresdner G, Gross B, Sumer SO, Sun Y, Jacobsen A, Sinha R, Larsson E, et al: Integrative analysis of complex cancer genomics and clinical profiles using the cBioPortal. Sci Signal 6: pl1, 2013.

25. Cerami E, Gao J, Dogrusoz U, Gross BE, Sumer SO, Aksoy BA, Jacobsen A, Byrne CJ, Heuer ML, Larsson E, et al: The cBio cancer genomics portal: An open platform for exploring multidimensional cancer genomics data. Cancer Discov 2: 401-404, 2012.

26. Remmele W and Schicketanz KH: Immunohistochemical determination of estrogen and progesterone receptor content in human breast cancer. Computer-assisted image analysis (QIC score) vs. subjective grading (IRS). Pathol Res Pract 189: 862-866, 1993.

27. Schiergens TS, Reu S, Neumann J, Renz BW, Niess H, Boeck S, Heinemann V, Bruns CJ, Jauch KW and Kleespies A: Histomorphologic and molecular phenotypes predict gemcitabine response and overall survival in adenocarcinoma of the ampulla of Vater. Surgery 158: 151-161, 2015.

28. Hsu HP, Shan YS, Jin YT, Lai MD and Lin PW: Loss of E-cadherin and beta-catenin is correlated with poor prognosis of ampullary neoplasms. J Surg Oncol 101: 356-362, 2010. 
29. Shan YS, Chen YL, Lai MD and Hsu HP: Nestin predicts a favorable prognosis in early ampullary adenocarcinoma and functions as a promoter of metastasis in advanced cancer. Oncol Rep 33: 40-48, 2015.

30. Piscuoglio S, Lehmann FS, Zlobec I, Tornillo L, Dietmaier W, Hartmann A, Wünsch PH, Sessa F, Rümmele P, Baumhoer D, et al: Effect of EpCAM, CD44, CD133 and CD166 expression on patient survival in tumours of the ampulla of Vater. J Clin Pathol 65: 140-145, 2012.

31. Rostain F, Hamza S, Drouillard A, Faivre J, Bouvier AM and Lepage C: Trends in incidence and management of cancer of the ampulla of Vater. World J Gastroenterol 20: 10144-10150, 2014.

32. Hsu HP, Yang TM, Hsieh YH, Shan YS and Lin PW: Predictors for patterns of failure after pancreaticoduodenectomy in ampullary cancer. Ann Surg Oncol 14: 50-60, 2007.

33. Goldstein SR, Yang GY, Curtis SK, Reuhl KR, Liu BC Mirvish SS, Newmark HL and Yang CS: Development of esophageal metaplasia and adenocarcinoma in a rat surgical model without the use of a carcinogen. Carcinogenesis 18: 2265-2270, 1997.

34. Pols TW, Noriega LG, Nomura M, Auwerx J and Schoonjans K: The bile acid membrane receptor TGR5: A valuable metabolic target. Dig Dis 29: 37-44, 2011.
35. Stepanov V, Stankov K and Mikov M: The bile acid membrane receptor TGR5: A novel pharmacological target in metabolic, inflammatory and neoplastic disorders. J Recept Signal Transduct Res 33: 213-223, 2013

36. Guo C, Su J, Li Z, Xiao R, Wen J, Li Y, Zhang M, Zhang X, Yu D, Huang W, et al: The G-protein-coupled bile acid receptor Gpbar1 (TGR5) suppresses gastric cancer cell proliferation and migration through antagonizing STAT3 signaling pathway. Oncotarget 6: 34402-34413, 2015.

37. Yasuda H, Hirata S, Inoue K, Mashima H, Ohnishi $H$ and Yoshiba M: Involvement of membrane-type bile acid receptor M-BAR/TGR5 in bile acid-induced activation of epidermal growth factor receptor and mitogen-activated protein kinases in gastric carcinoma cells. Biochem Biophys Res Commun 354: 154-159, 2007.

38. Jensen DD, Godfrey CB, Niklas C, Canals M, Kocan M, Poole DP, Murphy JE, Alemi F, Cottrell GS, Korbmacher C, et al: The bile acid receptor TGR5 does not interact with $\beta$-arrestins or traffic to endosomes but transmits sustained signals from plasma membrane rafts. J Biol Chem 288: 22942-22960, 2013 